\title{
Surgical management of squamous cell carcinoma arising in patients affected by epidermolysis bullosa: a comparative study
}

Dear Editors,

\section{INTRODUCTION}

Hereditary epidermolysis bullosa (EB) is a group of rare congenital diseases characterised by extreme epithelial fragility, which determines the formation of bullae and/or erosions either spontaneously or after local mechanical traumas. ${ }^{1}$ Both the skin and the mucosae are often involved. The severity of the disease is highly variable: while milder forms do not alter significantly the quality of life of the patients, more severe forms can be lethal at birth or even before. According to the depth of the lesions, EB is divided into three classes: simple, junctional, and dystrophic. ${ }^{2}$ Various inheritance patterns are possible, with the autosomal-recessive one being the most frequent. Junctional EB (JEB) in particular is generally caused by mutations in genes coding for proteins crucial for dermal-epidermal junction (DEJ) formation. Dystrophic EB (DEB), on the contrary, is mainly linked to mutations in the gene COL7A1, coding for collagen VII. In EB patients, especially in DEB and JEB variants, skin fragility leads to many possible complications and comorbidities. Apart from death during childhood due to either sepsis or respiratory failure, JEB and DEB are often burdened by ocular diseases, joint deformities, and a very high incidence of cutaneous squamous cell carcinoma (SCC). ${ }^{3,4}$ SCCs in EB patients generally arise in friction areas, more often in the extremities, where chronic blisters and scars are generally located. SCCs represent a big therapeutic challenge in the EB population: wound closure after surgical excision is in fact often difficult. ${ }^{5}$ If, on the one hand, primary suture in distal areas-often characterised by erosions and scars-is sometimes impossible, on the other hand, healthy donor sites for skin grafting are rarely available. Therefore, secondary intention wound healing is a very frequently used strategy for EB patients after surgical resections for oncological purposes. ${ }^{4}$ In this setting, the use of dermal matrices could find an important application. In fact, dermal scaffolds are proven to enhance and accelerate granulation tissue formation and to promote re-epithelization. ${ }^{6,7}$

The aim of our study was to compare the efficacy of a commonly used dermal substitute (Matriderm ${ }^{\circledR}$ ) and classical secondary-intention wound healing in determining wound bed vascularization and wound closure after surgery for SCC removal in EB patients.

\section{MATERIALS AND METHODS}

Two male patients affected by EB with invasive SCC of the extremities were included in the present study. The first patient (p1) was 32 years old and was affected by DEB. He presented with an invasive SCC of the ulnar margin of the left hand (see Figure 1A). The second patient (p2) on the contrary was a 49-year-old man, affected by JEB who referred to our centre for the presence of an invasive SCC of the right ankle (see Figure 1B). Both patients underwent surgical excision of the skin tumours. Then, while one patient (p1) underwent dermal substitute positioning, the other (p2) experienced second-intention wound closure (see Figure 1C,D). In both cases, transepidermal water loss and the infection risk were minimised through external dressings. Patients had two scheduled follow-up visits at day $15( \pm 5)$ and day $30( \pm 5)$ post-intervention (v1 and v2, respectively). During the follow-up, patients underwent not only clinical assessment but also microscopical evaluation of the wound bed after skin sample collection. Both classical histology with conventional haematoxylin and eosin and immunostaining for CD31 were performed at every timepoint.

\section{RESULTS}

Both patients had good clinical outcomes with complete re-epithelization at v2. However, re-epithelization was faster in the presence of dermal substitute, with better outcomes at v1, thus lowering the need of advanced 
dressings and shortening the time at high risk of infection (see Figure 1E-H). Moreover, minor scarring was developed after dermal substitute positioning, with a
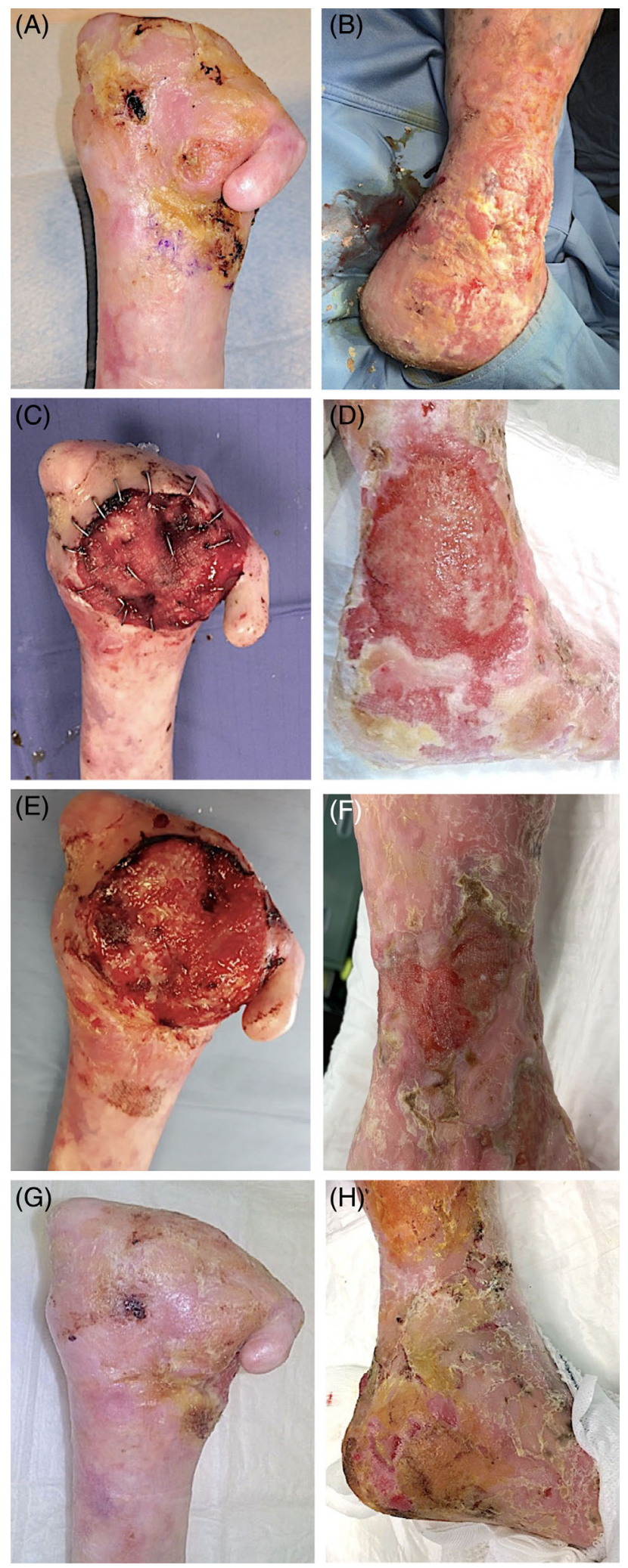

F I G URE 1 Legend on next column. score of 5 and 9, respectively, in patients 1 and 2 according to the Vancouver Scar Scale. A thicker epidermis was shown to be present in histological sections at v2 in the patient who was treated by Matriderm ${ }^{\circledR}$ positioning (Figure 2A,B). Revascularization of the wound bed occurred efficiently in both cases. In particular, in p1, vessels had a physiological distribution: in fact, CD31 + capillaries at v2 were mainly localised in the superficial and in the deep dermis, corresponding to the superficial and deep vascular dermal plexuses, with only sporadic anastomoses in the mid-dermis, as it happens under normal conditions. On the contrary, a hypervascularized dermis was evident in $\mathrm{p} 2$ at the same timepoint, as it often occurs in scar tissues, with redundant vessels, not restricted to vascular plexuses but diffused throughout the dermis, following the tensile strength of the scar.

\section{CONCLUSION}

In conclusion, patients affected by EB-especially in the junctional and dystrophic variants-have a 70-fold increase of SCC in areas of ulceration and scarring that often require demolitive surgery. ${ }^{8}$ In this setting, dermal matrices seem to improve significantly surgical outcomes in patients affected by EB.

$$
\begin{array}{r}
\text { Alessia Paganelli, } \mathrm{MD}^{1,2} \\
\text { Camilla Reggiani, } \mathrm{MD}^{1,2} \\
\text { Chiara Fiorentini, } \mathrm{MD}^{1} \\
\text { Mario Lando, } \mathrm{MD}^{3} \\
\text { Anna M. Cesinaro, } \mathrm{MD}^{4} \\
\text { Cristina Magnoni, MD, } \mathrm{PhD}^{1}
\end{array}
$$

${ }^{1}$ Surgical, Medical and Dental Department of Morphological Sciences related to Transplant, Oncology and Regenerative Medicine, University of Modena and Reggio Emilia, Italy

${ }^{2} \mathrm{PhD}$ Program in Clinical and Experimental Medicine, University of Modena and Reggio Emilia, Italy

${ }^{3}$ Department of Muscle-Skeletal Surgery, Hand and Microsurgery Division, Modena University Hospital, Italy

${ }^{4}$ Department of Pathological Anatomy, Modena University Hospital, Italy

FIG URE 1 Clinical pictures of the two EB patients before and after surgery. Patient 1 (p1): left column. Patient 2 (p2): right column. Panels A and B: p1 and p2 with squamous cells' carcinomas of the extremities before surgical intervention. Panels $\mathrm{C}$ and D: p1 and p2 3 days after surgery. Panels E and F: p1 and p2 15 days after surgery (visit 1, v1). Panels G and $\mathrm{H}$ : p1 and p2 30 days after surgery (v2) 

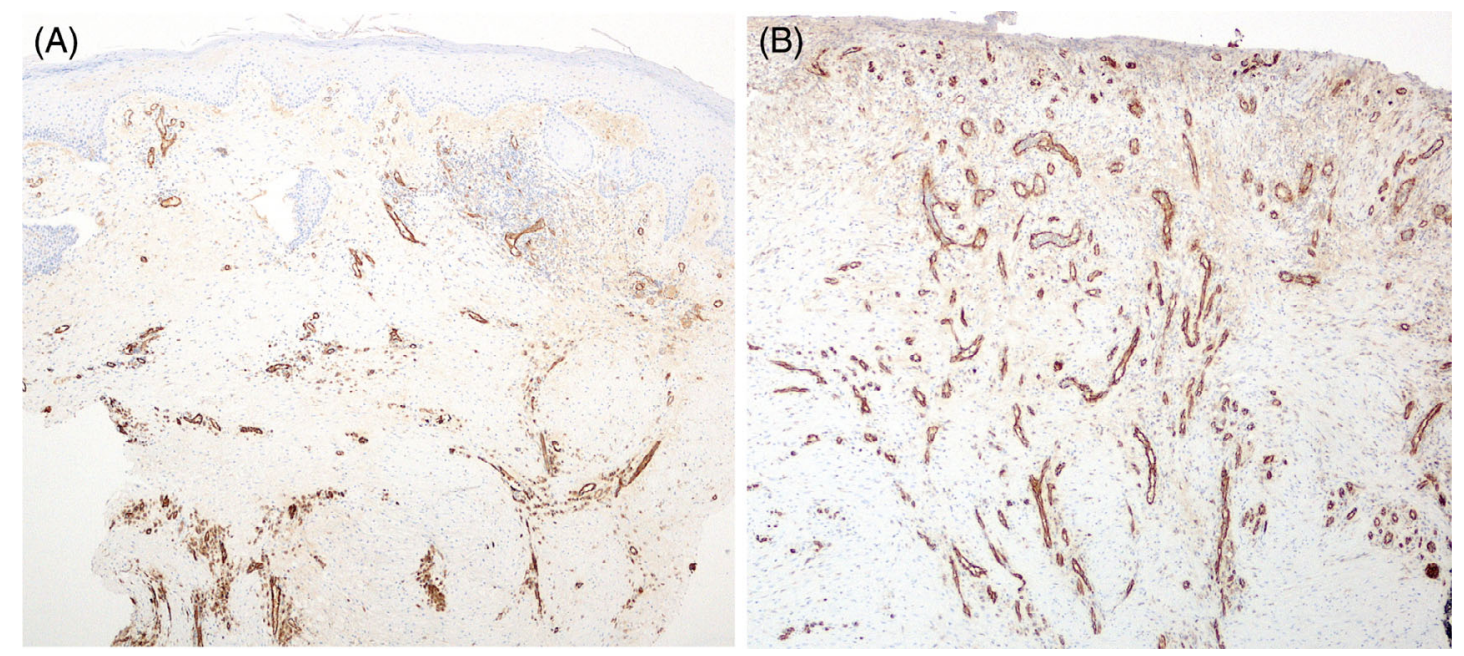

F I G U R E 2 CD31 staining for endotheliocytes while in p2 vessels were oriented linearly (panel B), following the tensile strength of the scar, a more physiological distribution of vascular structures throughout the dermis was observed in p1 (panel A)

Correspondence

Alessia Paganelli MD, University of Modena and Reggio Emilia, Dermatology Unit, via del Pozzo n. 71 Modena,

IT 41124.

E-mail: alessia.paganelli@gmail.com

\section{ORCID}

Alessia Paganelli @ https://orcid.org/0000-0002-0916-

7769

\section{REFERENCES}

1. El Hachem M, Giancristoforo S, Diociaiuti A. Inherited epidermolysis bullosa. G Ital Dermatol Venereol 2014;149(6): 651-662.

2. Fine J-D, Bruckner-Tuderman L, Eady RAJ, Bauer EA, Bauer JW, Has C, Heagerty A, Hintner H, Hovnanian A, Jonkman MF, Leigh I, Marinkovich MP, Martinez AE, McGrath JA, Mellerio JE, Moss C, Murrell DF, Shimizu H, Uitto J, Woodley D, Zambruno G. Inherited epidermolysis bullosa: updated recommendations on diagnosis and classification. J Am Acad Dermatol 2014;70(6):1103-1126.
3. Yamada M, Hatta N, Sogo K, Komura K, Hamaguchi Y, Takehara K. Management of squamous cell carcinoma in a patient with recessive-type epidermolysis bullosa dystrophica. Dermatol Surg 2004;30(11):1424-1429.

4. Rodríguez-Lojo R, Fernández-Jorge B, DE Andrés A, Peña C, García-Silva J. Wound closure by secondary intention is successful in the treatment of squamous cell carcinomas in recessive dystrophic epidermolysis bullosa. Eur J Dermatol 2011 Apr;21 (2):302-303.

5. Condorelli AG, Dellambra E, Logli E, Zambruno G, Castiglia D. Epidermolysis bullosa-associated squamous cell carcinoma: from pathogenesis to therapeutic perspectives. Int $\mathrm{J} \mathrm{Mol} \mathrm{Sci}$ 2019;20(22):5707.

6. Dai C, Shih S, Khachemoune A. Skin substitutes for acute and chronic wound healing: an updated review. J Dermatolog Treat 2018:1-33.

7. Kirsner RS, Bohn G, Driver VR, Mills JL, Nanney LB, Williams ML, et al. Human acellular dermal wound matrix: evidence and experience. Int Wound J 2015;12(6):646-654.

8. Cohn HI, Teng JMC. Advancement in management of epidermolysis bullosa. Curr Opin Pediatr 2016;28(4):507-516. 